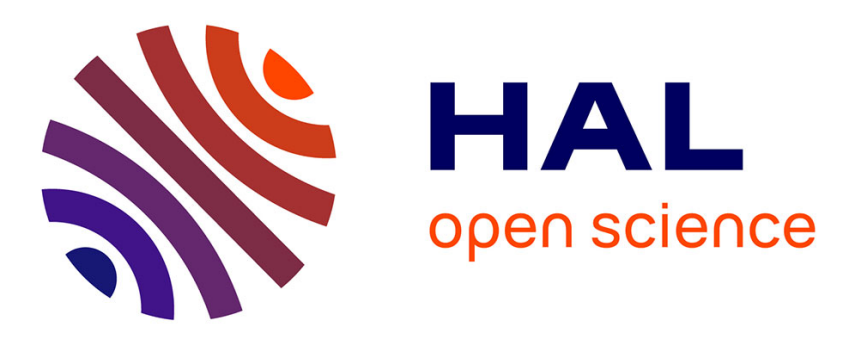

\title{
A virtual source pattern method for fluorescence tomography with structured light
}

Nicolas Ducros, Cosimo d'Andrea, Andrea Bassi, Gianluca Valentini, Simon Arridge

\section{- To cite this version:}

Nicolas Ducros, Cosimo d'Andrea, Andrea Bassi, Gianluca Valentini, Simon Arridge. A virtual source pattern method for fluorescence tomography with structured light. Physics in Medicine and Biology, 2012, 57 (12), pp.3811. 10.1088/0031-9155/57/12/3811 . hal-00874765

\section{HAL Id: hal-00874765 https://hal.science/hal-00874765}

Submitted on 18 Oct 2013

HAL is a multi-disciplinary open access archive for the deposit and dissemination of scientific research documents, whether they are published or not. The documents may come from teaching and research institutions in France or abroad, or from public or private research centers.
L'archive ouverte pluridisciplinaire HAL, est destinée au dépôt et à la diffusion de documents scientifiques de niveau recherche, publiés ou non, émanant des établissements d'enseignement et de recherche français ou étrangers, des laboratoires publics ou privés. 


\title{
Virtual source pattern method for fluorescence tomography with structured light
}

\author{
Nicolas Ducros ${ }^{1}$, Cosimo D'Andrea ${ }^{1,2}$, Andrea Bassi ${ }^{1}$, \\ Gianluca Valentini ${ }^{1}$, and Simon Arridge ${ }^{3}$ \\ ${ }^{1}$ Instituto di Fotonica e Nanotecnologie (IFN-CNR); Dipartimento di Fisica, \\ Politecnico di Milano, Piazza Leonardo da Vinci 32, I-20133 Milan, Italy. \\ 2 Italian Institute of Technology (IIT), Piazza Leonardo da Vinci 32, I-20133 \\ Milan, Italy. \\ ${ }^{3}$ Centre for Medical Image Computing, University College London, Malet \\ Place, London WC1E 6BT, United Kingdom. \\ E-mail: nicolas.ducros@polimi.it
}

\begin{abstract}
In order to reduce both acquisition and reconstruction times, illumination and detection in fluorescence diffuse optical tomography (FDOT) have recently evolved from a point-based to a pattern-based approach. The use of structured illumination, offering the ability to project any pattern of light onto the object, associated to the compression of the acquired fluorescence images has paved the way to a new generation of fast reconstruction algorithms for FDOT. However, the choice of the most appropriate set of source patterns is still an open problem.

Here, the use of typical source patterns is investigated on experimental data. Reconstructions of similar qualities are obtained for the different types of source patterns. We found that the performances of structured illumination are limited by the required positivity of the source patterns. To alleviate this problem, we introduce a novel method, namely the virtual source patterns method, which allows for considering any kind of patterns, e.g., with negative and complex intensities. This new method provides a significant increase of the contrast of the reconstruction and also a reduction of the reconstruction error, especially when virtual wavelet source patterns are considered.
\end{abstract}

Keywords: Fluorescence tomography, optical tomography, dimensionality reduction, image compression. 


\section{Introduction}

In the last years, the rapid development of specifically designed optical markers and technological improvements of imaging system, has fostered the field of optical molecular imaging (Cherry, 2004; Ntziachristos et al., 2003). This methodology allows one to follow, using longitudinal studies, functional processes and disease pathways at the molecular level in the living animal. The main advantages of optical techniques are represented by their high sensitivity, low invasiveness and low cost.

The main challenge of optical molecular imaging is to deal with thick tissues for which the images appear blurred due to scattering. Dealing with animal models, this aspect is particularly problematic for mice, whose dimensions and optical properties correspond to a fully diffusive regime, such that transmission imaging suffers from a lack of depth resolution. Fluorescence diffuse optical tomography (FDOT), also referred to as fluorescence molecular tomography (FMT), has been designed to solve these problems allowing to reconstruct the three-dimensional (3-D) fluorescence distribution in small samples (Milstein et al., 2003; Ntziachristos et al., 2005; Herv et al., 2007; Montet et al., 2007; Koenig et al., 2008; Kumar et al., 2008; Klose and Pschinger, 2011).

In classical FDOT, part of the sample surface is illuminated by a point source and the fluorescent light reemitted by the tissue is acquired by a detector (CCD cameras or photomultipliers via an optical fibre). Such a measurement is repeated by changing the input source position and/or rotating the sample. To improve the spatial resolution of the fluorescence reconstruction, a common strategy consists in using a large amount of illumination and detection points. However, due to the increase in the size of the data set, this strategy is not always numerically tractable. Moreover it leads to a significant increase of the acquisition time. To remain compatible with in vivo measurements, a trade off between acquisition/reconstruction times and reconstruction performances must be established.

In the last years, different research groups have begun to study the possibility to increase the speed of FDOT by reducing the data-set without sacrificing the reconstruction quality (Cuccia et al., 2005; Bassi et al., 2009; Konecky et al., 2009). To this end, it has been proposed to reduce the dimension of the measurement space by implementing two main concepts, often together. First, a series of works have capitalized on the low-pass filtering of the spatial frequencies of light propagating in highly scattering medium (Cuccia et al., 2005; Bassi et al., 2008). Fluorescent images obtained from biological tissue can be compressed to few coefficients with little degradation using image basis sets such as Fourier (D'Andrea et al., 2010; Ripoll, 2010) or wavelets (Rudge et al., 2010; Ducros et al., 2010b, 2011). Knowing the basis functions corresponding to the retained coefficients, referred to as detection patterns, a compressed version of the inverse problem can be numerically solved. This leads to a significant reduction in the reconstruction time. Analytical expressions for reconstruction with large data sets have also been developed (Markel et al., 2003; Wang et al., 2005) and successfully applied to experimental data (Panasyuk et al., 2008). However, the latter approach is limited to simple geometries such as slabs, cylinders, or spheres, for which symmetry properties are available (Markel and Schotland, 2004).

Second, it has been proposed to adopt patterns of illumination instead of point sources (Cuccia et al., 2005; Joshi et al., 2006a,b; Konecky et al., 2008; Lukic et al., 2009; Konecky et al., 2009; Dutta et al., 2010) with a view to reducing the number 
of illuminations. Indeed, few well-chosen patterns can be used to acquire as much information as a dense raster scanning of a point source (Lukic et al., 2009; Ducros et al., 2010a). A collateral advantage is that wide field illuminations allow one to project high light power on the sample without overcoming safety limits based on power density.

In this context, we have proposed to use both detection and illumination compression. In this way, fast 3-D reconstructions in arbitrary geometry, has been demonstrated with experimental data acquired from a slab medium (Ducros et al., 2010a) and from a cylinder (Ducros et al., 2011). Recently, a similar approach was used to perform tomographic measurement of small animals in time domain in Chen et al. (2010).

An open problem lies in the choice of the most appropriate set of illumination patterns to be projected onto the sample. Up to now different types of source patterns have been proposed. They can be categorized into five groups:

- Uniform or plane wave. It consists in illuminating the object with a spatially uniform pattern (Joshi et al., 2006b).

- ad hoc. Different type of patterns have been empirically tested. Among them, moving bars (Joshi et al., 2006b; Bélanger et al., 2010), scanning Gaussians and diffractive optics patterns (Joshi et al., 2006a), checkerboard Bélanger et al. (2010).

- Sinusoids. The homogeneous infinite slab being a purely low pass filter, the sinusoidal illumination patterns have been proposed to detect the presence of embedded inclusions. This natural candidate has been used for imaging purpose on the slab (Cuccia et al., 2005), but also for analytical (Konecky et al., 2009) and numerical (Bassi et al., 2009; D'Andrea et al., 2010; Ripoll, 2010) reconstruction in simple geometries.

- Wavelets bases. For more complex geometries wavelet bases have been proposed (Ducros et al., 2010a, 2011) for their well known compression ability.

- Optimal patterns. A fundamental step has been made by Dutta et al. (2010) who provided optimal source patterns for a slab, a cylinder, and a numerical phantom simulating the optical properties of animal models (Digimouse).

However, a comparative study among the different patterns is still missing. In particular, it remains unclear how the choice of the source pattern translates in terms of reconstruction quality when experimental data are considered.

The goal of this paper is twofold. The first goal is to experimentally compare the typically-used source patterns in term of reconstruction quality. In particular, it will be shown that even if different patterns are used the reconstruction quality is similar to that obtained with a uniform illumination. Since physically realisable source distributions are necessarily positive, a second goal of this paper is to introduce the virtual source patterns (VSP) method which is a general method able to determine the diffusive sample response to any negative or complex pattern and therefore to fully take advantage of the structured illumination. In Section 2, we first give the main equations formulating the forward problem of FDOT. Then, a method enabling to consider arbitrary patterns is presented. Last, the four steps of our reconstruction algorithm are described. In Section 3, we present five case studies. The first three case studies deal with actual sources patterns and the last two implement virtual source patterns. In Section 4, we discuss the reconstruction results that show a clear advantage in implementing the VSP method. Finally, our conclusions are reported in Section 5 . 
Table 1. Mathematical notations

\begin{tabular}{lll}
\hline Variable & Meaning & Dimension \\
\hline$I$ & Number of views & 1 \\
$J$ & Number of actual source patterns & 1 \\
$\tilde{J}$ & Number of virtual source patterns & 1 \\
$K$ & Number of retained components & 1 \\
$N$ & Number of voxels & 1 \\
$\Omega$ & Volume of the medium & $\mathbb{R}^{3}$ \\
$\partial \Omega$ & Surface of the medium & $\mathbb{R}^{2}$ \\
$\partial \Omega^{\mathrm{e}}$ & Illumination surface & $\mathbb{R}^{2}$ \\
$\partial \Omega^{\mathrm{m}}$ & Detection surface & $\mathbb{R}^{2}$ \\
$\Sigma^{\mathrm{e}}$ & Illumination plane & $\mathbb{R}^{2}$ \\
$\Sigma^{\mathrm{m}}$ & Detection plane & $\mathbb{R}^{2}$ \\
$\phi$ & Photon density & $\mathbb{C}(\Omega)$ \\
$\boldsymbol{d}$ & Detection pattern & $\mathbb{C}$ \\
$\boldsymbol{f}$ & Discrete fluorescence distribution & $\mathbb{R}^{N}$ \\
$\boldsymbol{m}$ & measured image & $\mathbb{R}^{N_{\mathrm{ccd}}}$ \\
$\tilde{\boldsymbol{m}}$ & virtually measured image & $\mathbb{C}^{N_{\mathrm{ccd}}}$ \\
$\boldsymbol{s}$ & source pattern & $\mathbb{R}_{+}^{N_{\mathrm{dmd}}}$ \\
$\tilde{\boldsymbol{s}}$ & virtual source pattern & $\mathbb{C} N_{\mathrm{dmd}}$ \\
$\mathcal{B}$ & Boundary condition operator & $\mathbb{C}(\Omega) \rightarrow \mathbb{C}(\Omega)$ \\
$\mathcal{M}$ & Measurement operator & $\mathbb{C}\left(\partial \Omega^{\mathrm{m}}\right) \rightarrow \mathbb{C}\left(\partial \Sigma^{\mathrm{m}}\right)$ \\
$\mathcal{P}$ & Propagation operator & $\mathbb{C}(\Omega) \rightarrow \mathbb{C}(\Omega)$ \\
$\mathcal{X}$ & Illumination operator & $\mathbb{C}\left(\partial \Sigma^{\mathrm{e}}\right) \rightarrow \mathbb{C}\left(\partial \Omega^{\mathrm{e}}\right)$ \\
\hline & & \\
\hline & &
\end{tabular}

\section{Method}

In this paper, italic letters $(a, \alpha$ or $A)$ indicate functions or constants. Vectors are denoted by lowercase bold italic letters ( $\boldsymbol{a}$ or $\boldsymbol{\alpha})$ and matrices by capital bold italic letters $(\boldsymbol{A}$ or $\boldsymbol{\Phi})$. We denote $\langle f, g\rangle_{\Omega}=\int_{\boldsymbol{r} \in \Omega} f(\boldsymbol{r}) \overline{g(\boldsymbol{r})} \mathrm{d} \boldsymbol{r}$ the inner product between two functions $f$ and $g, \mathcal{A}^{*}$ the adjoint of operator $\mathcal{A}$, and $\bar{x}$ the complex conjugate of $x$. A summary of the notation used throughout the paper is provided in table 1 .

\subsection{Forward Problem}

As depicted in figure 1, we consider a device, e.g. a digital micromirror device (DMD), able to illuminate a portion $\partial \Omega^{\mathrm{e}}$ of the surface $\partial \Omega$ of the medium $\Omega$ embedding a fluorescence distribution $f(\boldsymbol{r})$. The fluorescence light emitted from the surface $\partial \Omega^{m}$ is recorded by a CCD camera. The forward problem consists in determining the fluorescence image acquired by the CCD knowing the source pattern $s$ loaded on the DMD and the fluorescence distribution $f$.

Within the medium, the photon density $\phi\left(\mathrm{W} \cdot \mathrm{m}^{-2}\right)$ obeys the following coupled equations (Arridge and Schotland, 2009):

$$
\begin{array}{ll}
\mathcal{P}^{\mathrm{e}} \phi^{\mathrm{e}}=0 \text { in } \Omega & \text { and } \quad \mathcal{B}^{\mathrm{e}} \phi^{\mathrm{e}}=\mathcal{X} \boldsymbol{s} \text { on } \partial \Omega, \\
\mathcal{P}^{\mathrm{f}} \phi^{\mathrm{f}}=\phi^{\mathrm{e}} \eta \mu_{\mathrm{a}}^{\mathrm{f}} \text { in } \Omega & \text { and } \quad \mathcal{B}^{\mathrm{f}} \phi^{\mathrm{f}}=0 \text { on } \partial \Omega .
\end{array}
$$

where $\mathcal{P}$ is the propagation operator, $\mathcal{B}$ is an operator accounting for boundary conditions, and $\mathcal{X}$ is a mapping from the DMD plane $\Sigma^{\mathrm{e}}$ to the surface object $\partial \Omega^{\mathrm{e}}$. Equation (1a) describes the propagation of light at the excitation wavelength $\lambda_{\mathrm{e}}$ while 


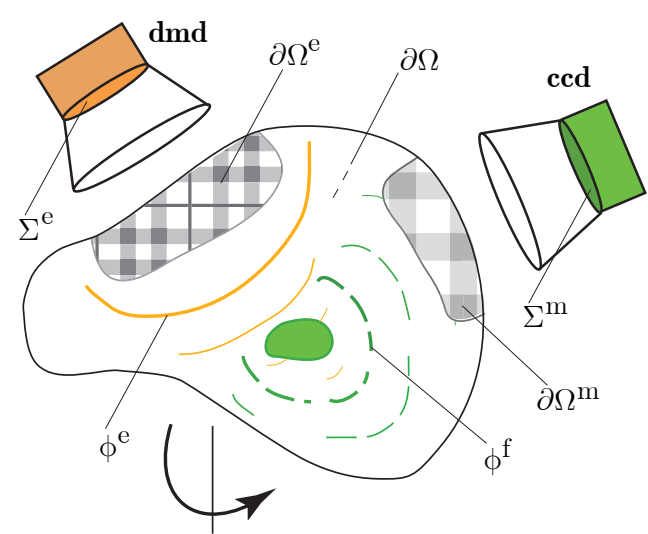

Figure 1. Principle of acquisition and notations

(1b) describes propagation at the fluorescence wavelength $\lambda_{\mathrm{f}}$. In (1b), the source term $\eta \mu_{\mathrm{a}}^{\mathrm{f}} \phi^{\mathrm{e}}\left(\mathrm{W} . \mathrm{m}^{-3}\right)$ represents the part of the photons absorbed at $\lambda_{\mathrm{e}}$ that are remitted at $\lambda_{\mathrm{f}}$, with $\eta$ the quantum yield of the fluorescent marker and $\mu_{\mathrm{a}}^{\mathrm{f}}$ its absorption coefficient. In what follows, we denote $f=\eta \mu_{\mathrm{a}}^{\mathrm{f}}$ the fluorescence distribution, which is to be recovered. In this paper, we consider the diffusion approximation together with partial boundary conditions, which leads to (Ishimaru, 1977)

$$
\begin{gathered}
\mathcal{P}=-\nabla[D(\boldsymbol{r}) \nabla]+\mu_{\mathrm{a}}(\boldsymbol{r}), \quad \text { and } \\
\mathcal{B}=\mathcal{I}-A D(\boldsymbol{r}) \hat{\boldsymbol{n}} \cdot \nabla,
\end{gathered}
$$

where $D$ is the diffusion constant of the medium and $\mu_{\mathrm{a}}$ its absorption coefficient. In $(2 b), \mathcal{I}$ stands for the identity operator, $A$ is a constant that depends on the index mismatch, and $\hat{\boldsymbol{n}}$ indicates the outward-pointing normal to the surface $\partial \Omega$.

The fluorescence image acquired by the CCD is $\boldsymbol{m}=\mathcal{M}\left[\phi^{\mathrm{f}}\right]$ where $\mathcal{M}$ is a mapping from the object surface $\partial \Omega^{\mathrm{m}}$ to the CCD plane $\Sigma^{\mathrm{m}}$. In our approach we compress the acquired image

$$
\boldsymbol{m} \simeq \hat{\boldsymbol{m}}=\sum_{k=1}^{K} \hat{m}_{k} \boldsymbol{d}_{k}
$$

where the $\boldsymbol{d}_{k}$ are the basis of the image compression method; in analogy to the source compression basis used herein, we consider the image compression basis vectors as detection patterns.

Given a fluorescence distribution $f(\boldsymbol{r})$, the components $\hat{m}_{k}$ can be obtained by means of an integral operator with kernel $w$. Mathematically:

$$
\hat{m}_{k}=\left\langle w\left(s, \boldsymbol{d}_{k}\right), f\right\rangle_{\Omega}
$$

where the integration kernel $w$ only depends on the source and detection pattern $s$ and $\boldsymbol{d}_{k}$. It can be shown that (see appendix A for proof)

$$
w\left(\boldsymbol{s}, \boldsymbol{d}_{k}\right)=\mathcal{G}^{\mathrm{e}}[\boldsymbol{u}] \overline{\mathcal{G}^{\mathrm{f} *}\left[\boldsymbol{v}_{k}\right]},
$$


where $\mathcal{G}^{\mathrm{e}}\left(\right.$ resp. $\left.\mathcal{G}^{\mathrm{f}}\right)$ is the Green's operator for (1a) (resp. (1b)), $\boldsymbol{u}=\mathcal{X}[\boldsymbol{s}]$ is the source pattern mapped onto surface $\partial \Omega^{\mathrm{e}}$, and $\boldsymbol{v}_{k}$ is the adjoint detection pattern defined on surface $\partial \Omega^{\mathrm{m}}$. The adjoint detection pattern is defined by

$$
\boldsymbol{v}_{k}=\mathcal{M}^{*}\left[\boldsymbol{d}_{k}\right]
$$

In a general problem, the object is rotated and multiple-illumination acquisitions are performed. We denote $I$ the number of rotation angles and $J$ the number of source patterns that are projected onto the object for each view. Such a process results in the acquisition of a set of $I J$ images. Each of them is compressed to $K$ components, which leads to a total number of $K_{\text {tot }}=I J K$ image components. Discretizing the volume $\Omega$ in $N$ voxels, the forward problem given in (4) simplifies to the discrete product

$$
\hat{\boldsymbol{m}}=\boldsymbol{W} \boldsymbol{f} .
$$

where $\hat{\boldsymbol{m}} \in \mathbb{C}^{K_{\text {tot }}}$ is the measurement vector containing the retained image components, $\boldsymbol{W} \in \mathbb{C}^{K_{\mathrm{tot}} \times N}$ is a matrix referred to as the weight matrix, and $\boldsymbol{f} \in \mathbb{R}^{N}$ is the discrete concentration vector. We denote by $\boldsymbol{w}^{i, j, k}$ the discretised kernel of (5) for the $k^{t h}$ detection pattern of the $j^{\text {th }}$ source and $i^{\text {th }}$ rotation, with $k$ the fastest varying index, followed by $j$, and with $i$ the slowest varying index. Equation (7) can be written:

$$
\left(\begin{array}{c}
\hat{m}_{1,1,1} \\
\vdots \\
\hat{m}_{1,1, k} \\
\vdots \\
\hat{m}_{1, j, k} \\
\vdots \\
\hat{m}_{i, j, k} \\
\vdots \\
\hat{m}_{I, J, K}
\end{array}\right)=\left(\begin{array}{ccccc}
w_{1}^{1,1,1} & \ldots & w_{n}^{1,1,1} & \ldots & w_{N}^{1,1,1} \\
\vdots & & & & \vdots \\
w_{1}^{1,1, k} & \ldots & w_{n}^{1,1, k} & \ldots & w_{N}^{1,1, k} \\
\vdots & & & & \vdots \\
w_{1}^{1, j, k} & \ldots & w_{n}^{1, j, k} & \ldots & w_{N}^{1, j, k} \\
\vdots & & & & \vdots \\
w_{1}^{i, j, k} & \ldots & w_{n}^{i, j, k} & \ldots & w_{N}^{i, j, k} \\
\vdots & & & & \vdots \\
w_{1}^{I, J, K} & \ldots & w_{n}^{I, J, K} & \ldots & w_{N}^{I, J, K}
\end{array}\right) \cdot\left(\begin{array}{c}
f_{1} \\
\vdots \\
f_{n} \\
\vdots \\
f_{N}
\end{array}\right)
$$

\subsection{Virtual source patterns method}

Here we describe a simple approach, namely the virtual source pattern (VSP) method, that offers the possibility to exploit the response of the diffusive sample to any negative or complex pattern, although only patterns with positive intensities can be projected on the sample. The method capitalize on the linearity of the forward problem.

Let $\tilde{\boldsymbol{S}}=\left[\begin{array}{lll}\tilde{\boldsymbol{s}}_{1} \ldots & \tilde{\boldsymbol{s}}_{\tilde{J}}\end{array}\right]$ represent the $\tilde{J}$ (desired) virtual source patterns. Our approach consist in finding $J$ actual source patterns $\boldsymbol{S}=\left[\boldsymbol{s}_{1} \ldots s_{J}\right]$ and a $\tilde{J} \times J$ (possibly complex) matrix $\boldsymbol{T}$ such that

$$
\tilde{\boldsymbol{S}}^{\mathrm{T}}=\boldsymbol{T} \boldsymbol{S}^{\mathrm{T}} .
$$

The matrix $\boldsymbol{T}$ is referred to as transformation matrix. As a consequence of linearity, the same transformation can be applied to the actual measurements $\boldsymbol{M}=\left[\boldsymbol{m}_{1} \ldots \boldsymbol{m}_{J}\right]$ so as to get the virtual measurements $\tilde{\boldsymbol{M}}=\left[\tilde{\boldsymbol{m}}_{1} \ldots \tilde{\boldsymbol{m}}_{\tilde{J}}\right]$. The virtual images would have been measured if the virtual patterns $\tilde{\boldsymbol{S}}$ were projected onto the medium. Formally:

$$
\tilde{\boldsymbol{M}}^{\mathrm{T}}=\boldsymbol{T} \boldsymbol{M}^{\mathrm{T}}
$$




\subsection{Virtual wavelet patterns}

In this section, we describe how to generate wavelet patterns by means of the virtual source method. The reader can refer to the work of Mallat (1989) for a general discussion on wavelet bases.

The wavelet functions (mother wavelets) can be derived from the so-called scaling functions (father wavelets). In dimension 2, at a given resolution, the i) $M$ vertical wavelets $\left\{\boldsymbol{q}^{\mathrm{V}}\right\}$, ii) $M$ horizontal wavelets $\left\{\boldsymbol{q}^{\mathrm{H}}\right\}$, and iii) $M$ diagonal wavelets $\left\{\boldsymbol{q}^{\mathrm{D}}\right\}$ can be constructed from the $4 M$ scaling functions $\{\boldsymbol{p}\}$ taken at a higher resolution. Hence, to obtain virtual wavelet patterns $\widetilde{\boldsymbol{S}}$ in (9), the actual source patterns $\boldsymbol{S}$ can be chosen as scaling functions and the transformation matrix $\boldsymbol{T}$ can be constructed accordingly. We choose:

$$
\tilde{\boldsymbol{S}}=\left(\begin{array}{c}
\boldsymbol{q}_{1}^{\mathrm{V}} \\
\boldsymbol{q}_{1}^{\mathrm{H}} \\
\boldsymbol{q}_{1}^{\mathrm{D}} \\
\vdots \\
\boldsymbol{q}_{M}^{\mathrm{V}} \\
\boldsymbol{q}_{M}^{\mathrm{H}} \\
\boldsymbol{q}_{M}^{\mathrm{D}}
\end{array}\right) \quad \boldsymbol{T}=\left(\begin{array}{ccc}
t_{1,1}^{\mathrm{V}} & \ldots & t_{1,4 M}^{\mathrm{V}} \\
t_{1,1}^{\mathrm{H}} & \ldots & t_{1,4 M}^{\mathrm{H}} \\
t_{1,1}^{\mathrm{D}} & \ldots & t_{1,4 M}^{\mathrm{D}} \\
\vdots & & \vdots \\
t_{M, 1}^{\mathrm{V}} & \ldots & t_{M, 4 M}^{\mathrm{V}} \\
t_{M, 1}^{\mathrm{H}} & \ldots & t_{M, 4 M}^{\mathrm{H}} \\
t_{M, 1}^{\mathrm{D}} & \ldots & t_{M, 4 M}^{\mathrm{D}}
\end{array}\right) \quad \boldsymbol{S}=\left(\begin{array}{c}
\boldsymbol{p}_{1} \\
\vdots \\
\boldsymbol{p}_{4 M}
\end{array}\right)
$$

As detailed in Appendix A.2, each row of $\boldsymbol{T}$ contains the coefficients of a $2 \mathrm{D}$ vertical, horizontal or diagonal high-pass filter, reshaped into a $4 M \times 1$ vector. Hence, any DC component added to the scaling functions $\boldsymbol{S}$ cancels out after multiplication with $\boldsymbol{T}$. This guarantees that any wavelet pattern can be generated even if the corresponding scaling functions contain negative value. The technical details to computing $\boldsymbol{T}$ are reported in appendix Appendix A.2.

\subsection{FDOT reconstruction algorithm}

The sketch of our algorithm is presented in figure 2. The different steps are detailed below. We denote $\boldsymbol{m}_{i, j}$ the fluorescence image measured at the $i$ th view after illumination of the object with the $j$ th source pattern $\boldsymbol{s}_{j}$.

Step 1 The $I \tilde{J}$ virtual fluorescence images $\tilde{\boldsymbol{m}}_{i, j}$ are calculated from the $I J$ fluorescence images $\boldsymbol{m}_{i, j}$ according to (10). The same way, the $\tilde{J}$ virtual source patterns $\tilde{\boldsymbol{s}}_{j}$ are calculated from the $J$ actual source patterns $\boldsymbol{s}_{j}$ according to (9). The virtual source patterns are mapped from the DMD plane $\Sigma^{\mathrm{e}}$ onto the object surface $\partial \Omega_{i}^{\mathrm{e}}$ and the resulting $\tilde{\boldsymbol{u}}_{i, j}=\mathcal{X}_{i} \tilde{\boldsymbol{s}}_{j}$ are concatenated to form the virtual source matrix $\tilde{\boldsymbol{U}}=\left[\tilde{\boldsymbol{u}}_{1,1} \ldots \tilde{\boldsymbol{u}}_{i, j} \ldots \tilde{\boldsymbol{u}}_{I, \tilde{J}}\right] \in \mathbb{C}^{N \times I \tilde{J}}$.

Step 2 Each of the $I \tilde{J}$ virtual fluorescence image $\tilde{\boldsymbol{m}}_{i, j}$ is wavelet transformed and retaining the $K_{i, j}$ (absolute value) largest components leads to the compressed image

$$
\hat{\boldsymbol{m}}_{i, j}=\sum_{k=1}^{K_{i, j}} \hat{m}_{i, j, k} \boldsymbol{d}_{k}
$$

The retained components $\hat{m}_{i, j, k}$ are stacked into the vector $\hat{\boldsymbol{m}} \in \mathbb{C}^{K_{\text {tot }}}$. In this paper, the detection patterns $\boldsymbol{d}_{k}$ are chosen from a Daubechie 4 basis.

Next, the adjoint detection patterns $\boldsymbol{v}_{i, j, k}$, defined on the object surface $\partial \Omega_{i}^{m}$, 


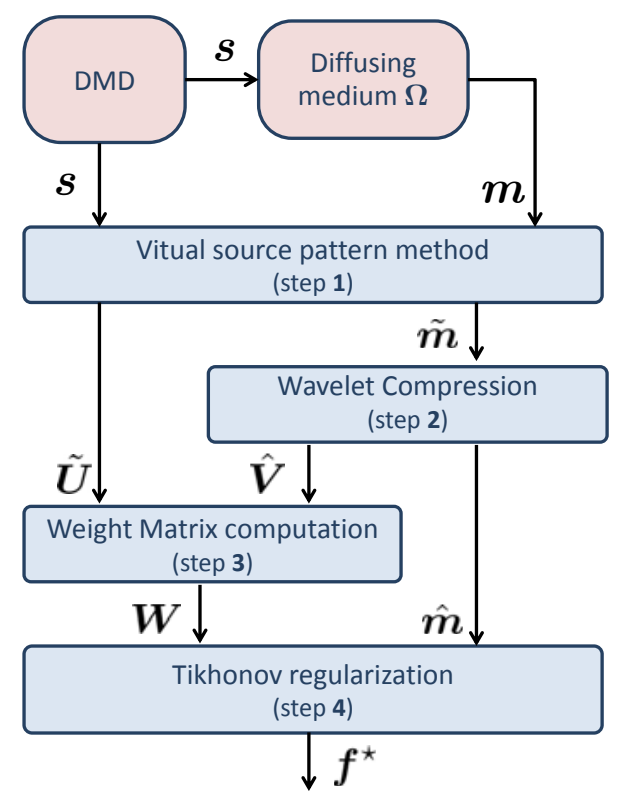

Figure 2. Sketch of the FDOT reconstruction algorithm.

are calculated from the detection patterns $\boldsymbol{d}_{k}$, defined on the CCD plane $\Sigma^{\mathrm{m}}$. According to (6), we have $\boldsymbol{v}_{i, k}=\mathcal{M}_{i}^{*}\left[\boldsymbol{d}_{k}\right]$. For our problem, it is sufficient to consider the $L$ non-redundant detection patterns $\hat{\boldsymbol{v}}_{\ell}$, with $L<K_{\text {tot }}$, which reduces the computational cost of using multiple source patterns. Finally, all the adjoint detection patterns are concatenated into a single matrix $\hat{\boldsymbol{V}}=\left[\hat{\boldsymbol{v}}_{1} \ldots \hat{\boldsymbol{v}}_{\ell} \ldots \hat{\boldsymbol{v}}_{L}\right] \in$ $\mathbb{C}^{N \times L}$

Step 3 The computation of the weight matrix $\boldsymbol{W} \in \mathbb{C}^{K_{\text {tot }} \times N}$ requires the calculation of $\boldsymbol{\varphi}_{i, j} \in \mathbb{C}^{N}$ and $\boldsymbol{\psi}_{i, k} \in \mathbb{C}^{N}$ that are the vectorised versions of $\mathcal{G}^{\mathrm{e}}[\boldsymbol{u}]$ and $\mathcal{G}^{\mathrm{f} *}\left[\boldsymbol{v}_{k}\right]$ in (5), respectively. The source density $\boldsymbol{\varphi}_{i, j}$ is needed for all couples $(i, j)$, while the adjoint detection density $\boldsymbol{\psi}_{i, k}$ must be obtained for any couple $(i, k)$. This may be done in one shot inverting the matrix equations

$$
\boldsymbol{P}^{\mathrm{e}} \boldsymbol{\Phi}=\tilde{\boldsymbol{U}} \quad \text { and } \quad \boldsymbol{P}^{\mathrm{f}} \hat{\boldsymbol{\Psi}}=\hat{\boldsymbol{V}}
$$

The matrix $\boldsymbol{\Phi}=\left[\boldsymbol{\varphi}_{1,1} \ldots \boldsymbol{\varphi}_{i, j} \ldots \boldsymbol{\varphi}_{I, \tilde{J}}\right] \in \mathbb{C}^{N \times I \tilde{J}}$ contains the source densities $\boldsymbol{\varphi}_{i, j}$ and the matrix $\hat{\boldsymbol{\Psi}}=\left[\hat{\boldsymbol{\psi}}_{1} \ldots \hat{\boldsymbol{\psi}}_{\ell} \ldots \hat{\boldsymbol{\psi}}_{L}\right] \in \mathbb{C}^{N \times L}$ contains the non-redundant adjoint detection densities $\hat{\boldsymbol{\psi}}_{\ell}$. Any vector $\boldsymbol{\psi}_{i, k}$ can be obtained by picking up the appropriate $\hat{\boldsymbol{\psi}}_{\ell}$ in $\hat{\boldsymbol{\Psi}}$. In the latter equation, $\boldsymbol{P}^{\mathrm{e}}$ and $\boldsymbol{P}^{\mathrm{f}}$ are two $N \times N$ sparse matrices that result from the discretization of the propagation operator $\mathcal{P}^{\mathrm{e}}$ and $\mathcal{P}^{\mathrm{f}}$ and account for the boundary conditions. Here, $\boldsymbol{P}^{\mathrm{e}}$ and $\boldsymbol{P}^{\mathrm{f}}$ have been computed thanks to the Matlab TOAST package (Schweiger and Arridge, 2010). The weight matrix $\boldsymbol{W}$ is finally built up row by row, according to (5). Precisely, the row of $\boldsymbol{W}$ corresponding to the triplet $(i, j, k)$ is given by

$$
\boldsymbol{w}_{i, j, k}=\boldsymbol{\varphi}_{i, j} \circ \overline{\boldsymbol{\psi}_{i, k}}
$$

where $\circ$ stands for the entrywise (Hadamard) product. 
Step 4 The inverse problem in FDOT is well-known to be ill-posed and requires regularization to ensure the stability of the solution $f^{\star}$ in the presence of noise. Here, the inverse problem is efficiently solved considering the underdetermined Tikhonov solution

$$
\boldsymbol{f}^{\star}=\boldsymbol{W}^{\mathrm{T}}\left(\boldsymbol{W} \boldsymbol{W}^{\mathrm{T}}+\alpha \boldsymbol{I}\right)^{-1} \hat{\boldsymbol{m}}
$$

where $\alpha$ is the regularization parameter that enforces the stability of the solution. The procedure for choosing its value is detailed in Section 2.6.

\subsection{Sample and Experimental set-up}

We consider a cylindrical phantom mimicking the dimensions and optical properties of the mouse. The phantom is an epoxy resin cylinder of radius $20 \mathrm{~mm}$ and height 45 $\mathrm{mm}$. Its optical properties at $\lambda^{\mathrm{e}}=630 \mathrm{~nm}$ have been measured with a time-resolved spectroscopy system (Bassi et al., 2007) and the following values have been obtained: $\mu_{\mathrm{a}}=0.022 \mathrm{~mm}^{-1} \mu_{\mathrm{s}}^{\prime}=1.35 \mathrm{~mm}^{-1}$. The geometry of the fluorescent inclusions are represented at figure $3 \mathrm{~b}$.

The experimental set-up has been reported in detail in D'Andrea et al. (2010). It is based on three main components: illumination device, detection system and rotational stage sample holder. Light is emitted by a He:Ne laser source (05LHP991, Melles Griot) at wavelength of $633 \mathrm{~nm}$ with optical power of about $10 \mathrm{~mW}$ coupled to an optical fiber guide (LLG0538-4, Thorlabs). Then, the light is focused by a lens and, through a total internal reflection prism, is delivered to a Digital Micromirror Device (DMD) (Discovery 1100 - ALP1, Vialux). DMD consists of a matrix of titlable mirrors $(720 \times 521)$, whose reflecting elements can be independently controlled. By uploading the image of the desidered spatial pattern, the arrangement of the tiltable mirrors and the light beam reflected by the DMD change accordingly. Through the control of the duty cycle, patterns with a dynamic range of 8 bits can be generated by the DMD. Finally, the pattern is magnified and projected on the cylindrical phantom by means of an a projection lens. The illumination area $\partial \Omega^{\mathrm{e}}$ is a rectangle of width $\ell_{\mathrm{x}}=13 \mathrm{~mm}$ and height $\ell_{\mathrm{y}}=26 \mathrm{~mm}, 2.5 \mathrm{~mm}$ apart from the top of the cylinder. The sample holder is mounted on a motorized rotational stage in order to perform multiple view measurements.

The light exiting the opposite side of the cylindrical phantom is imaged by an objective lens $(f=50 \mathrm{~mm}, f \#=2.8$, Nikon Co. $)$ on a low noise high sensitivity 16 bit CCD camera (Versarray 512, Princeton Instruments,) cooled to $-40^{\circ} \mathrm{C}$. The detection area $\partial \Omega^{\mathrm{m}}$ is a rectangle of width $16 \mathrm{~mm}$ and height $32 \mathrm{~mm}$. The illumination and detection area are depicted in figure 3a. In order to select the fluorescence signal, a combination of an interference filter (XF 3076, Omega) and a high pass filter RG695 , Schott) is placed in front of the objective lens. The signal at the excitation wavelength can be acquired by removing the filters. The system is placed in a lightproof cabinet in order to prevent stray light. Particular attention has been devoted to the automatization of the measurements system, which is fully computer controlled, in order to reduce the acquisition time.

\subsection{Comparison of the reconstructions}

In order to fairly compare the reconstructions obtained from different types and numbers of illumination patterns, the choice of the regularization parameter is crucial. The proposed approach is inspired by Ducros et al. (2009), where the 


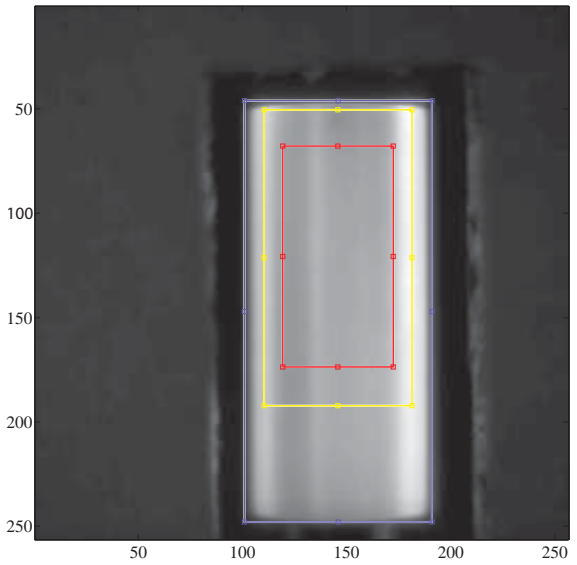

(a)

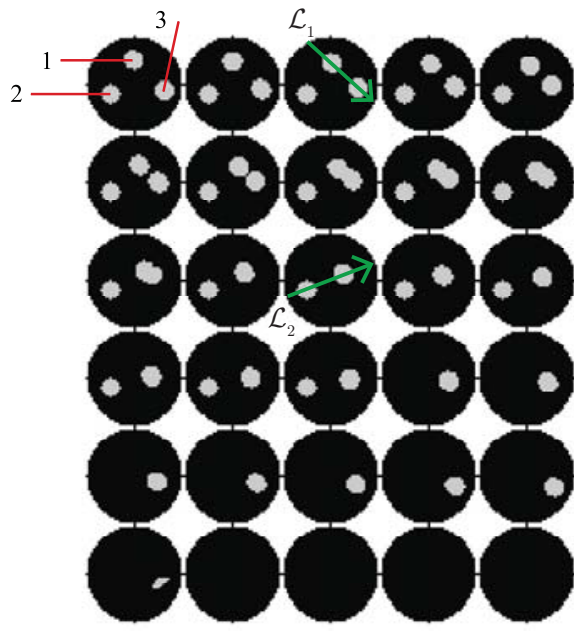

(b)

Figure 3. a) Source surface $\partial \Omega^{\mathrm{e}}\left(13 \times 26 \mathrm{~mm}^{2}\right.$, red line $)$ and detection surface $\partial \Omega^{\mathrm{m}}\left(16 \times 32 \mathrm{~mm}^{2}\right.$, yellow line $)$ superimposed on the cylinder. b) Cylindrical phantom (height $45 \mathrm{~mm}$, diameter $20 \mathrm{~mm}$ ). The three inclusions are cylinders with radius $2 \mathrm{~mm}$. Thirty slices are displayed from $z=-9.5 \mathrm{~mm}$ to $z=19.5$ $\mathrm{mm}$. The fluorophore concentration is about $10^{-4} \mathrm{M}$ of Nile Blue in Ethylene Glycol. The concentration has been chosen in order to achieve a peak signal of about $5 \times 10^{4}$ electrons per pixel in the camera sensor.

regularization parameter is set by imposing the reconstruction variability. Here, the regularization parameter is set by maximizing the contrast-to-noise ratio (CNR) of the reconstructed fluorescence distribution $f^{\star}$. The CNR is a figure of merit that indicates how well structures of interest are rendered in a reconstruction (Song et al., 2004; Baritaux et al., 2010). It is defined by

$$
C N R=\frac{\mu_{\text {roi }}-\mu_{\text {back }}}{\left(w_{\text {roi }} \sigma_{\text {roi }}^{2}+w_{\text {back }} \sigma_{\text {back }}^{2}\right)^{1 / 2}}
$$

where $\mu_{\text {roi }}\left(\mu_{\text {back }}\right)$ and $\sigma_{\text {roi }}\left(\sigma_{\text {back }}\right)$ are respectively the average and standard deviation of $\boldsymbol{f}^{\star}$ in the ROI (background). The weights $w_{\text {roi }}$ and $w_{\text {back }}$ are the volume ratios $w_{\text {roi }}=V_{\text {roi }} / V_{\text {tot }}$ and $w_{\text {back }}=V_{\text {back }} / V_{\text {tot }}$. Here, the region of interest (ROI) refers to the volume occupied by the fluorescence inclusions and the background refers to the rest of the volume.

In addition to the CNR, we consider other two figures of merit, namely the contrast resolution and the reconstruction error. The contrast resolution $\mathcal{C}$ is defined by

$$
\mathcal{C}=\frac{\mu_{\text {roi }}-\mu_{\text {back }}}{\mu_{\text {roi }}+\mu_{\text {back }}}
$$

It measures the ability to tell the difference between the ROI and the background in terms of mean values. The higher the contrast resolution, the better.

Finally, we consider the reconstruction error $\mathcal{E}_{r}$. Expressed in decibels, the following 

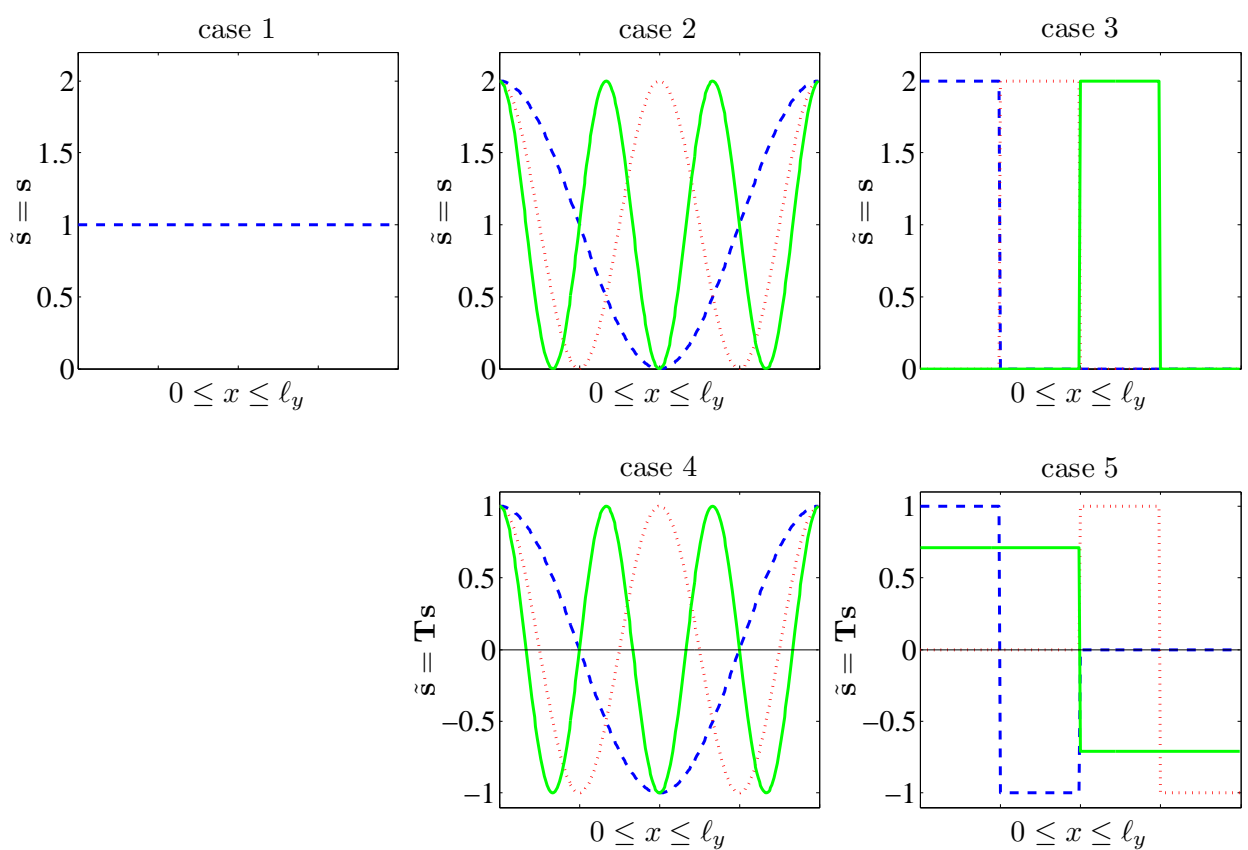

Figure 4. Some of the source patterns considered in the five cases.

definition is retained:

$$
\mathcal{E}_{r}(\mathrm{~dB})=20 \log \left\|\boldsymbol{f}^{\star} \frac{\mu_{\text {roi }}^{\text {true }}}{\mu_{\text {roi }}}-\boldsymbol{f}_{\text {true }}\right\|-20 \log \left\|\boldsymbol{f}_{\text {true }}\right\| .
$$

where $\mu_{\text {roi }}^{\text {true }}$ is the average of $\boldsymbol{f}_{\text {true }}$ in the ROI. The smaller the reconstruction error, the better.

\section{Results}

We present five experiments for five different types of source patterns. The first three experiments implement real source patterns while the last two implement virtual source patterns. The profiles of some source patterns are displayed for each experiment in figure 4 . The five experiments have been performed in the same conditions. Every source pattern has been projected onto the the cylindrical phantom for $\Delta t=35 \mathrm{~s}$. Sixteen rotation angles from $0^{\circ}$ to $337.5^{\circ}$ have been considered. For objective comparison among reconstruction qualities, the three performance metrics are calculated for each experiment and reported in table 2. Some reconstruction profiles for the five experiments are provided at figure 10. Another important aspect is the amount of time required to perform FDOT. In table 3 both acquisition and reconstruction times are reported for each experiment. 


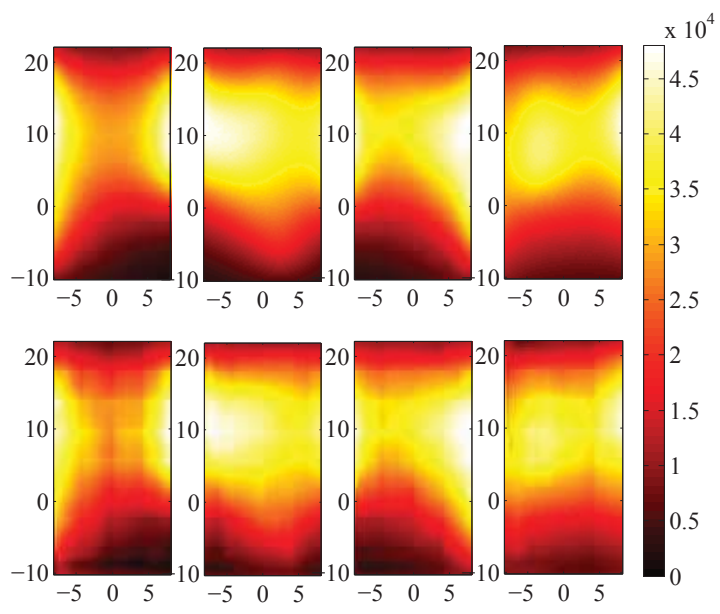

Figure 5. Fluorescence images acquired at $\theta=0^{\circ}, 90^{\circ}, 180^{\circ}, 270^{\circ}$, from right to left. A uniform illumination pattern is considered. Original images are represented in the top row while the compressed images are represented in the bottom row. The colorbar indicates the number of counts.

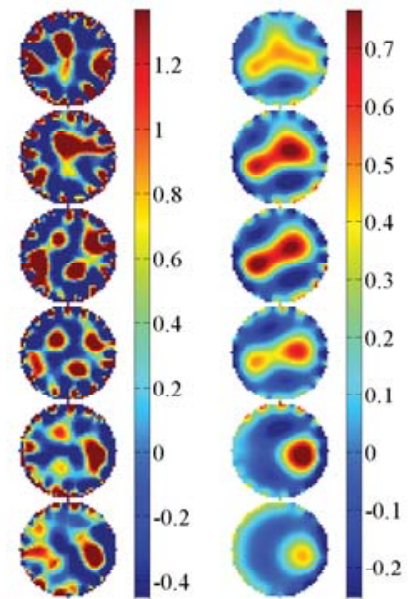

(a)

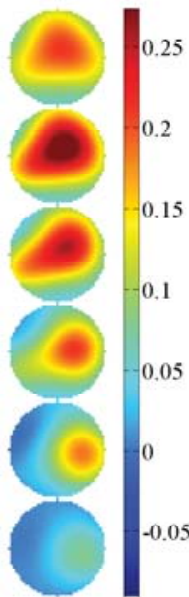

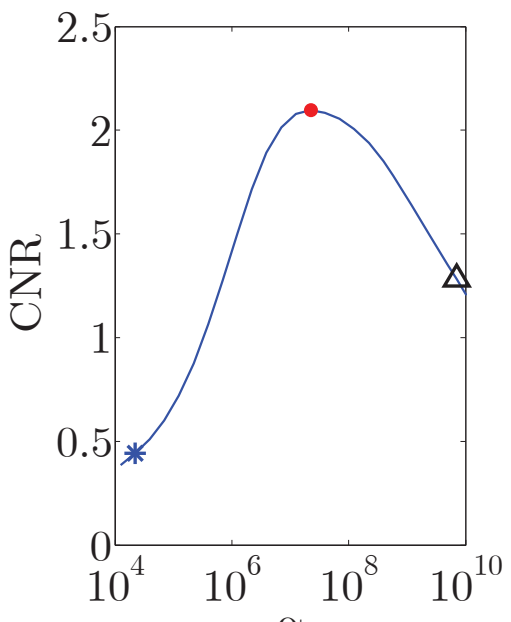

(b)

Figure 6. a) Reconstructions obtained for three different regularization parameters. Six slices are displayed from $z=17.5 \mathrm{~mm}$ to $z=-7.5 \mathrm{~mm}$. Central column: optimal regularization parameter $\alpha_{\text {opt }}$ as defined in Section 2.6; Left-hand column: under-regularized situation for which $\alpha=\alpha_{\text {under }}<\alpha_{\text {opt }}$, ; Right-hand column: over-regularized situation for which $\alpha=\alpha_{\text {over }}>\alpha_{\text {opt }}$. The maximum and minimum value of each colorbar have been set to $1.5 \mu_{\text {roi }}$ and $-0.5 \mu_{\text {roi }}$, respectively. b) Graph of the CNR of the reconstruction with respect to the regularization parameter $\alpha$. The blue asterisk indicates $\alpha_{\text {under }}$, the red dot $\alpha_{o p t}$, and the black triangle $\alpha_{o v e r}$. 


\subsection{Case 1 - Uniform illumination}

The first experiment is performed loading onto the DMD a constant image whose intensity level is set to the maximum value (255). This experiment is considered as the reference experiment. First, because it enables for measuring the highest signal in transmission. Indeed, the zero spatial frequency is the less attenuated one (see for example Bassi et al. (2007)). Second, since a DMD intrinsically cuts light off, this experiment benefits from the highest amount of light available.

The fluorescence images experimentally acquired are depicted in figure 5 . The choice of the regularization parameter follows the rule given in Section 2.6. In figure 6a we present some reconstruction slices corresponding to three different regularization parameters: a small $\alpha$, the optimal $\alpha$, and a large $\alpha$. The graph of the CNR with respect to the regularization parameter is given in figure $6 \mathrm{~b}$. The whole set of reconstruction slices for the optimal reconstruction parameter is depicted in figure 7a.

\subsection{Case 2 - Sinusoids}

In this experiment, we considered sinusoidal patterns of the form $\cos 2 \pi\left(n_{x} x / \ell_{x}+\right.$ $\left.n_{y} y / \ell_{y}\right)+1$, where $n_{x}$ and $n_{y}$ are the numbers of cycles along $x$ and $y$, respectively. In diffuse optics, the considered media behave as low-pass filters. Though source patterns of increasing spatial frequencies could increase the spatial resolution of reconstructions, such frequencies are increasingly attenuated and cannot be experimentally measured. Thus, a common strategy consists in considering few low frequency patterns. In this case, patterns up to 1 cycle along $x$ (the smallest dimension) and up to 3 cycles along $y$ (the largest dimension) are regarded. Precisely, a set of 8 bi-dimensional frequencies is considered: $n_{x} \in\{0,1\}$ and $n_{y} \in\{0,1,2,3\}$. Note that this experiment includes the uniform pattern of case 1 , i.e., $\left(n_{x}, n_{y}\right)=(0,0)$. Note also that, apart from this pattern, all the other patterns lead to an illumination of half the amount of total light. The resulting reconstructed fluorescence distribution is presented in figure $7 \mathrm{~b}$.

\subsection{Case 3 - Haar scaling functions}

In this experiment, we consider the 2-D scaling functions of a wavelet basis. It is well known that such functions can provide a multiresolution approximation of images (Unser and Blu, 2003). Since there is generally no closed form for these functions, they are more easily obtained from the inverse wavelet transform. Let $\boldsymbol{A s}$ denotes the wavelet transform of $s$ at a given resolution. The matrix $\boldsymbol{A}$ may be written $\boldsymbol{A}=\left[\boldsymbol{A}_{\mathrm{a}}, \boldsymbol{A}_{\mathrm{d}}\right]$ where $\boldsymbol{A}_{\mathrm{a}} \boldsymbol{s}$ provides an approximation of $\boldsymbol{s}$, while $\boldsymbol{A}_{\mathrm{d}} \boldsymbol{s}$ give the details of $\boldsymbol{s}$. Henceforth, the scaling patterns may be simply obtained as $\boldsymbol{s}=\boldsymbol{A}_{\mathrm{a}}^{-1} \hat{\boldsymbol{s}}$ where $\hat{\boldsymbol{s}}$ is a unit vector chosen from the natural basis $\left\{\boldsymbol{e}_{i}\right\}$.

In this experiment, we consider the Haar wavelet. At the resolution we consider, the patterns simplify to height black images containing a white square of side $\ell_{\mathrm{x}} / 2=\ell_{\mathrm{y}} / 4$. The reconstructed fluorescence distribution obtained from eight Haar scaling patterns is presented at figure $7 \mathrm{c}$.

\subsection{Case 4-Phasor methods}

The phasor method has been extensively used for demodulation in communications systems (Carlson et al., 2002) where it is known under square-law detection. Referred to as phase shifting technique, it has been a method of choice in profilometry 
(Srinivasan et al., 1984) and was successfully applied in confocal microscopy (Neil et al., 1997). In diffuse optics, it was first introduced by Cuccia et al. (2005) for filtering out the DC component in the measured images. The phasor method consists in i)projecting onto the medium a set of $P$ shifted source patterns $s[p]=$ $\cos 2 \pi\left(n_{x} x / \ell_{x}+n_{y} y / \ell_{y}+p / P\right)+1$, with $p \in\{0, P-1\}, p / P$ being the phase shift and ii) post processing the resulting $P$ images $\boldsymbol{m}$ to obtain a demodulated image $\tilde{\boldsymbol{m}}=\sum_{p} \boldsymbol{m}[p] \exp (\mathrm{i} 2 \pi p / P)$.

From the VSP point of view, the phasor method can be interpreted as the use of some actual source patterns $s$ to gain the response to a virtual source pattern $\tilde{\boldsymbol{s}}=\exp \left[\mathrm{i} 2 \pi\left(n_{x} x / \ell_{x}+n_{y} y / \ell_{y}\right)\right]$. Note the virtual source pattern no longer contain additive DC component. For a given pair of frequencies $\left(n_{x}, n_{y}\right)$ and choosing $P=3$, the transformation matrix defined in Section 2.2 simplifies to:

$$
\boldsymbol{T}=\left(\begin{array}{ccc}
1 & -\frac{1}{2} & -\frac{1}{2} \\
0 & 1 & -1
\end{array}\right)
$$

The patterns used in experiment 2 , with $n_{x} \in\{0,1\}$ and $n_{y} \in\{0,1,2,3\}$, are used together with three phase shifts $p \in\{0,1,2\}$, which results in a set of twenty-four actual source patterns. It is worth noting that after processing the measurements not only the real part, but also the imaginary part of the virtual fluorescence image are available.

The reconstruction of the fluorescence distribution obtained from the phasor method is available in figure $9 \mathrm{a}$.

\subsection{Case 5 - Virtual Haar wavelets}

While scaling functions are used in case 3, wavelet functions are desired in this experiment. Wavelet functions possess a higher frequency content than scaling functions and provide details (at different resolution level) in image compression. Implementing such patterns requires the VSP method since wavelets contain negative values. Interestingly, wavelet functions at a given resolution level are easily obtained from scaling functions at a finer resolution level (see Section 2.3). Capitalizing on this property, the measurements of case 3 can serve in this experiment to get the response to Haar wavelet patterns at a lower resolution. Considering the two filters $\boldsymbol{h}=\frac{1}{\sqrt{2}}[1,1]^{\mathrm{T}}$ and $\boldsymbol{g}=\frac{1}{\sqrt{2}}[1,-1]^{\mathrm{T}}$ and following the four step described in Section Appendix A.2 leads to the following transformation matrix:

$$
\boldsymbol{T}=\frac{1}{2}\left(\begin{array}{cccccccc}
1 & 1 & 0 & 0 & -1 & -1 & 0 & 0 \\
1 & -1 & 0 & 0 & 1 & -1 & 0 & 0 \\
1 & -1 & 0 & 0 & -1 & 1 & 0 & 0 \\
0 & 0 & 1 & 1 & 0 & 0 & -1 & -1 \\
0 & 0 & 1 & -1 & 0 & 0 & 1 & -1 \\
0 & 0 & 1 & -1 & 0 & 0 & -1 & 1
\end{array}\right)
$$

The virtual source patterns, i.e., the columns of $\tilde{\boldsymbol{S}}=\boldsymbol{T} \boldsymbol{S}$ are displayed in figure 8 together with the associated virtual measurements.

The reconstruction of the fluorescence distribution from the virtual Haar wavelets is available in figure $9 \mathrm{~b}$. 


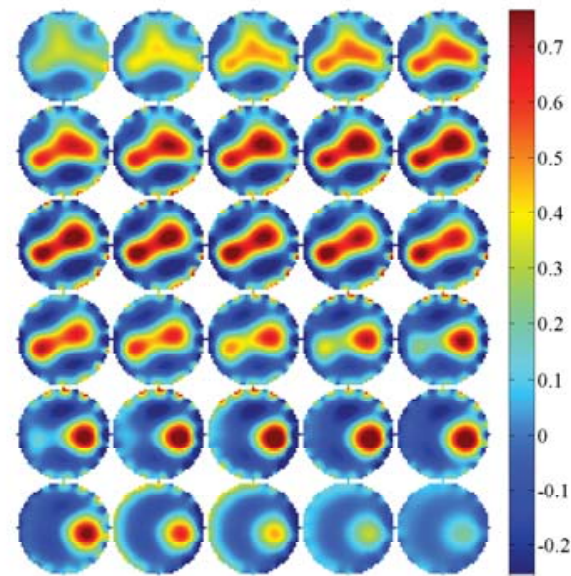

(a)

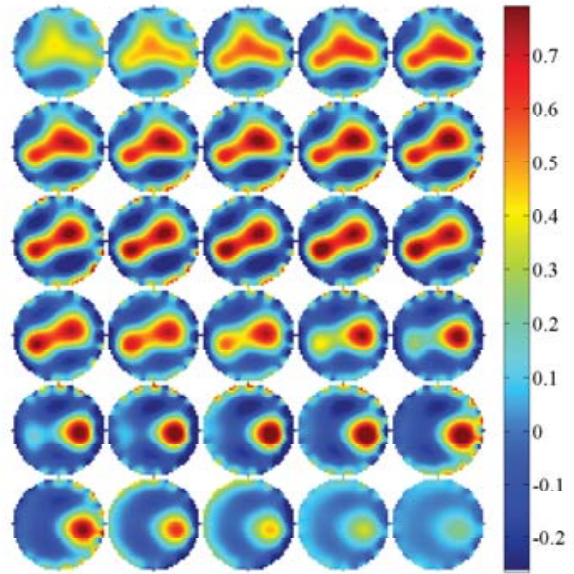

(b)

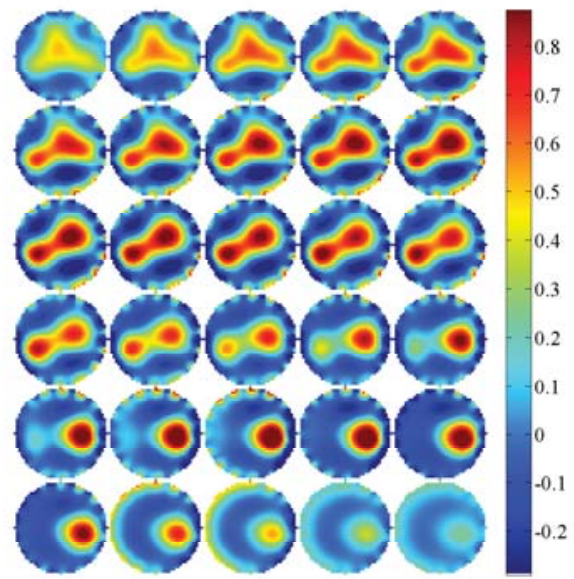

(c)

Figure 7. Reconstructions from actual illumination patterns: a) Plane wave (case 1); b) Sinusoids (case 2); c) Wavelet scaling functions (case 3). For each reconstruction, thirty slices are displayed from $z=19.5 \mathrm{~mm}$ to $z=-9.5 \mathrm{~mm}$. Reconstructions are given in arbitrary units. For fair comparison between the different reconstructions, the maximum and minimum value of each colorbar have been set to $1.5 \mu_{\text {roi }}$ and $-0.5 \mu_{\text {roi }}$, respectively.

Table 2. Performance metrics for the different illumination patterns

\begin{tabular}{cccc}
\hline Case & $C N R$ & $\mathcal{C}$ & $\mathcal{E}_{r}(\mathrm{~dB})$ \\
\hline 1 & 2.10 & 0.77 & -17.2 \\
2 & 2.09 & 0.77 & -18.3 \\
3 & 2.13 & 0.78 & -18.1 \\
4 & 2.49 & 0.83 & -18.6 \\
5 & 2.76 & 0.87 & -19.0 \\
\hline
\end{tabular}



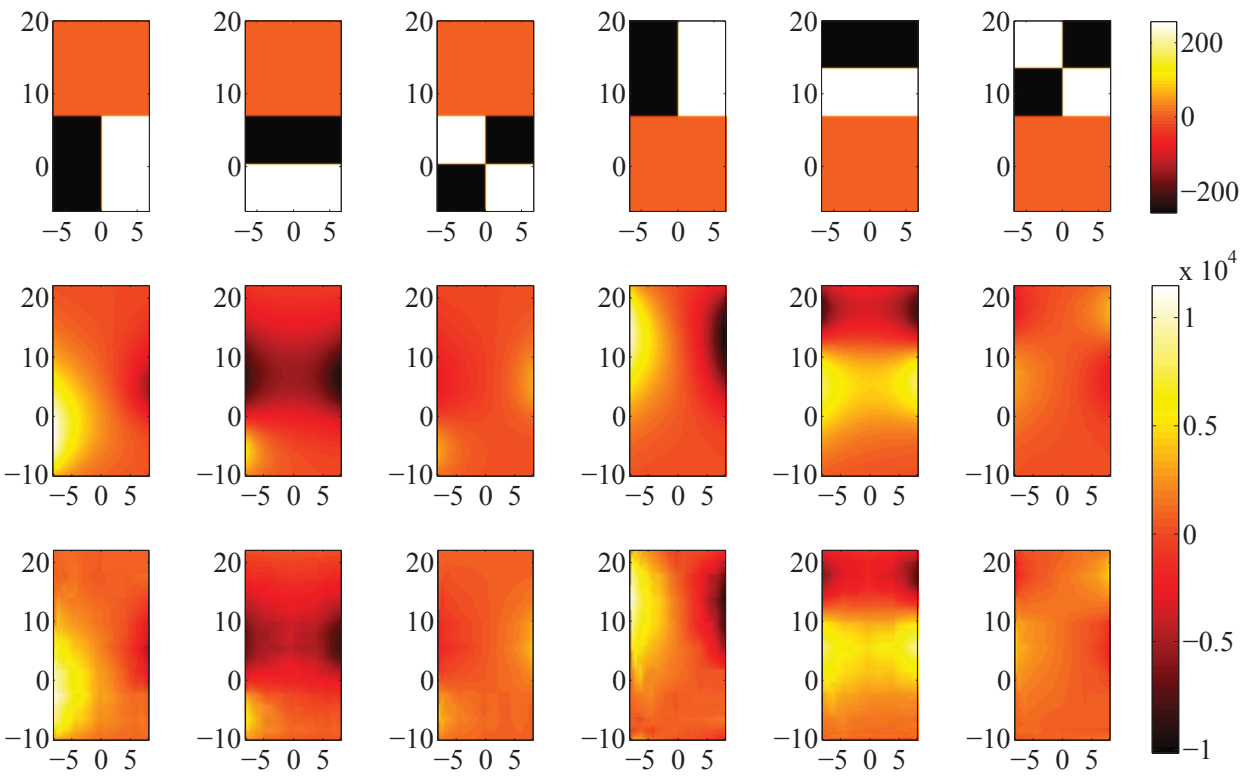

Figure 8. Acquisitions using the virtual illumination patterns of case 5. First row, virtual illumination patterns $\tilde{\boldsymbol{s}}$ on the DMD. The colorbar indicates gray levels. Second row, virtual fluorescence images $\tilde{\boldsymbol{m}}$ on the CCD. Third row, virtual fluorescence images after compression $\hat{\boldsymbol{m}}$. The colorbar indicates the numbers of counts.

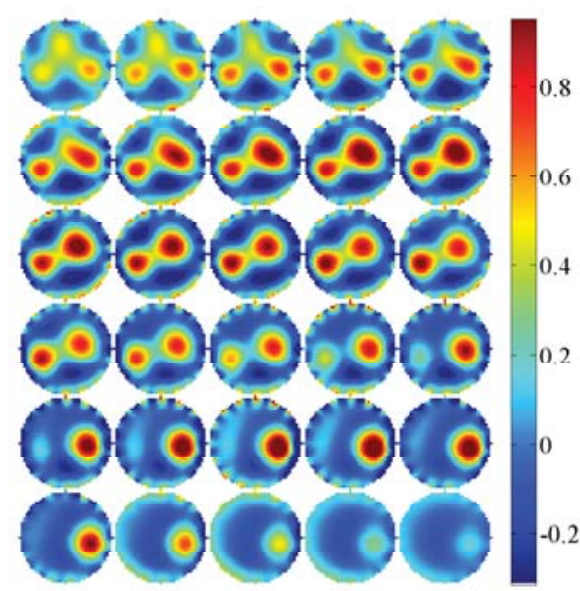

(a)

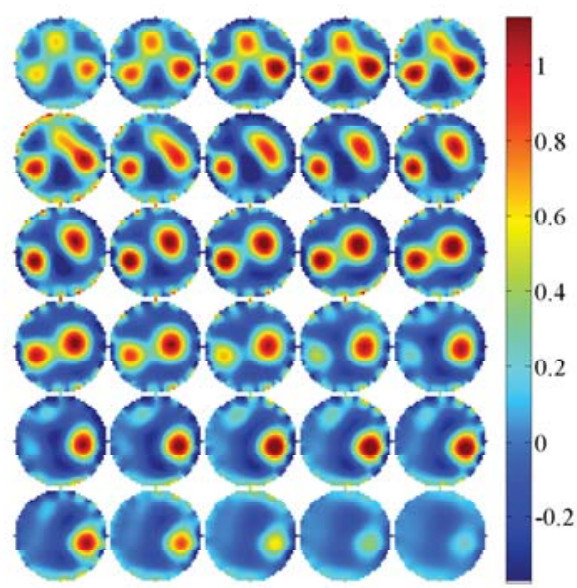

(b)

Figure 9. Reconstructions from virtual illumination patterns. a) sinusoid by means of the phasor method (case 4); b) wavelets functions (case 5). For each reconstruction, thirty slices are displayed from $z=19.5 \mathrm{~mm}$ to $z=-9.5 \mathrm{~mm}$. Reconstructions are given in arbitrary units. For fair comparison between the different reconstructions, the maximum and minimum value of each colorbar have been set to $1.5 \mu_{\text {roi }}$ and $-0.5 \mu_{\text {roi }}$, respectively. 


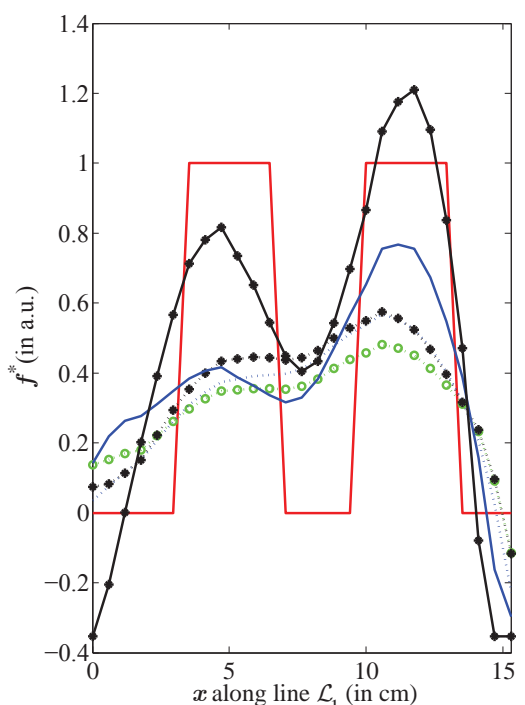

(a)

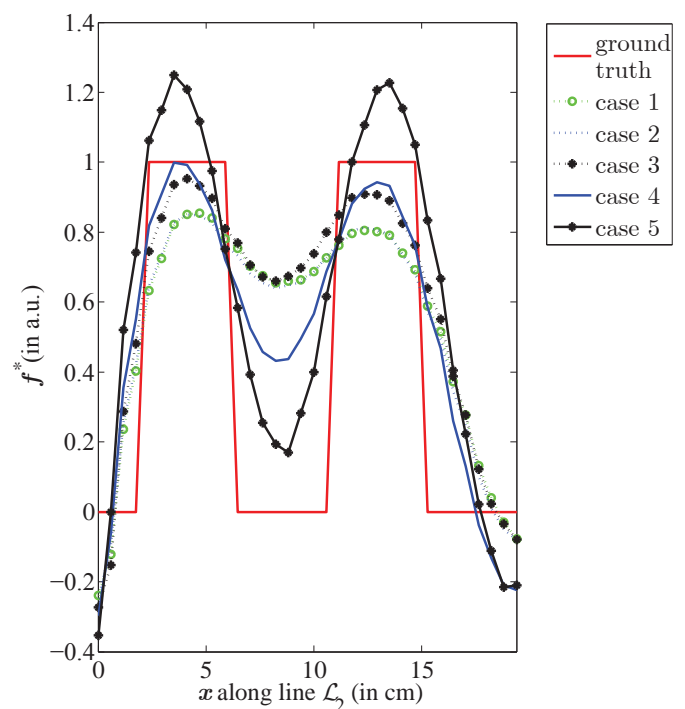

(b)

Figure 10. Profiles of reconstruction for the cases 1 to 5 . a) Reconstruction profiles along line $\mathcal{L}_{1}$, i.e., through inclusions 1 and 3 at $z=17.5 \mathrm{~mm}$. b) Reconstruction profiles along line $\mathcal{L}_{2}$, i.e., through inclusions 2 and 3 at $z=7.5$ mm. The two lines $\mathcal{L}_{1}$ and $\mathcal{L}_{2}$ are defined at figure $3 \mathrm{~b}$.

\section{Discussion}

Using only a uniform illumination, the overall quality of the reconstruction is quite good since the contours of the three inclusions can be recovered. Inclusion 2 is reconstructed with a correct localization in the $x y$ plane as well as along $z$. However, the reconstruction of inclusions 1 and 3 is of lower quality, especially in top slices down to $z=9.5 \mathrm{~mm}$, where inclusion 1 joins inclusion 3 . Reconstructed fluorescence is observed to vanish in the first top slices (from $z=19.5 \mathrm{~mm}$ to $z=17.5 \mathrm{~mm}$, in particular). The latter effect is a typical problem that can be attributed to the vicinity of boundary. It must be outlined that the top slices also suffer from receiving a limited amount of light since the illumination area starts $3 \mathrm{~mm}$ underneath the cylinder summit.

The reconstructions obtained from cases 1, 2, and 3 indicate the performance of the real source patterns. First of all, note that only one source pattern is considered in case 1 while height source patterns are considered in both cases 2 and 3 . Hence, the reconstruction quality is expected to be higher in case studies 2 and 3 than in case 1 (at an extra cost in terms of acquisition and computation times). By visual inspection of the reconstruction slices displayed in figure 7 , the benefit of using 8 source patterns appears limited. To get a better picture of the situation, it is informative to refer to the figures of merit reported in table 2 and to the reconstruction profiles of figure 10. An improvement is found with a diminution of the reconstruction error for both case studies 2 and 3 . While case 3 also exhibits a slight improvement in terms of CNR and contrast, case 2 stands at a CNR of 0.77 and decreases in terms of contrast.

Regarding now the two cases implementing the VSP method, it can be seen that the reconstruction quality is greatly improved with respect to the other three cases. 
Table 3. Acquisition and reconstruction times (in sec.) for the different case studies

\begin{tabular}{ccccccccc}
\hline Case & $J$ & Acquisition time (s) & $\tilde{J}$ & $L$ & \multicolumn{4}{c}{ Reconstruction time (s) } \\
& & & & & Step 2 & Step 3 & Step 4 & Total \\
\hline 1 & 1 & 35 & 1 & 1024 & 6.1 & 19 & 2 & 28 \\
2 & 8 & 280 & 8 & 1189 & 6.5 & 44 & 54 & 110 \\
3 & 8 & 280 & 8 & 1352 & 6.7 & 44 & 54 & 112 \\
4 & 24 & 840 & 16 & 1705 & 8.5 & 93 & 206 & 329 \\
5 & 8 & 280 & 6 & 2220 & 9.7 & 54 & 30 & 97 \\
\hline
\end{tabular}

Indeed, the best reconstruction obtained with actual source patterns, namely case 3 , is outperformed by both VSP reconstructions of case studies 4 and 5 (compare figure 7a to figure $9 \mathrm{a}$ and $\mathrm{b}$ and the profiles at figure 10). In particular, inclusion 1 is nicely individuated. Moreover, in spite of the lack of illumination light, the first three slices show little blurring. These visual observations are confirmed by the figures of merits reported in table 2. Among the two VSP implementations, the wavelet approach offers the best performance, whether in terms of CNR, contrast, or reconstruction error.

Very similar reconstruction qualities have been obtained from different types of actual source patterns. However, the VSP method results in a better reconstruction quality. For a given set of fluorescence images, the interest of combining real images to form virtual ones prior to reconstructing is demonstrated (compare case 2 to case 4 and also case 3 to case 5). A key feature of the VSP method lies in its ability to remove the DC components in the fluorescence images. This advantage is shown comparing case studies 1,2 , and 4 . First, note that each pattern of case 2 contains a DC component plus an AC component. Hence, the similarity of reconstruction 1 and 2 can indicate that the DC components in case 2 overwhelm the potentially useful AC components. This is confirmed by the results of case 4 that show a significant improvement of the reconstruction quality in the absence of the DC component. It must be outlined that removing the DC component is likely to be further profitable in experimental scenarios since it also permits to cancel out undesirable light. To capitalize on this important feature, a satisfying transformation matrix must highpass filter the actual fluorescence images. Hence, the coefficient of the columns of $\boldsymbol{T}$ must satisfy

$$
\sum_{j=1}^{J} t_{\tilde{\jmath}, j}=0, \quad \forall \tilde{\jmath} \in\{1, . ., \tilde{J}\},
$$

where the $t_{\tilde{\jmath}, j}$ 's are the matrix elements of $\boldsymbol{T}$.

The superiority of the VSP method is also clearly shown comparing cases 3 and 5 , that exploit the same data set. Note that the transformation matrix of case 5 (see (20)) is chosen to satisfy the previous condition (21). To further test the importance of removing DC components, we have also built the virtual wavelet patterns of case 5 choosing a transformation matrix that does not verify condition (21). This results in a poor reconstruction quality (results not shown), which support our interpretation. Although it exploits fewer patterns, the virtual wavelet approach outperforms the phasor method. An interpretation is that the phasor method, which works in the Fourier space, only exploits the frequency resolution of the source patterns and it is 
known that high frequencies are increasingly damped. On the other hand, the wavelet approach offers a trade-off between the spatial and frequency resolution of the source patterns. In this sense, the wavelet approach provide a trade-off between the pointsource approach and the phasor-method approach.

Another important aspect is the noise amplification. The virtual fluorescence images are by construction noisier than the actual fluorescence images they results from. Despite this, the reconstruction quality is improved using virtual patterns, which indicates the applicability of the method on real data.

While satisfying condition (21), there are many ways to choose $\boldsymbol{T}$ and $\boldsymbol{S}$ so as to get the desired $\tilde{\boldsymbol{S}}$. An important aspect of this choice is the cost of the VSP method in terms of extra measurements, which can be indicated through the ratio $\tilde{J} / J$. The most straightforward approach for obtaining a negative pattern $s$ is to separate its positive part $\boldsymbol{s}^{+}$from its negative part $\boldsymbol{s}^{-}$and to consider $\tilde{\boldsymbol{s}}=\boldsymbol{s}^{+}-\boldsymbol{s}^{-}$, which leads to $\tilde{J} / J=1 / 2$. The phasor method provides a more efficient approach for sinusoids with a ratio $\tilde{J} / J$ of $2 / 3>1 / 2$. In Section 2.3 , a method is given that enables for building $3 M$ wavelets from $4 M$ scaling functions. With this approach, we have: $\tilde{J} / J=3 / 4>2 / 3$, which is a better ratio than for phasor method. Note that even if the scaling functions contain negative values, the approach of Section 2.3 can still be used provided an appropriate background is added to the scaling functions.

The speed of our FDOT algorithm can be evaluated by means of table 3 . We limit the discussion to the case 4 and 5 for which the gain of reconstruction quality is substantial. Since the acquisition time is proportional to the number of real source patterns $\tilde{J}$, the phasor method requires an acquisition 3 times longer than the virtual wavelet approach. As far as the reconstruction time is concerned, the reduction of the set of detection patterns at step 3 allows for a great speed-up of the algorithm and the use of different source patterns for a given view comes at a limited extra cost. Anyway, the wavelet approach is about three times faster than the phasor method.

\section{Conclusions}

This work addresses the problem of the choice of the source patterns in fluorescence optical tomography considering experimental measurements. We have shown that different types of actual source patterns, which are limited to positive intensities, provides very similar reconstruction qualities. A positive pattern can be considered as a uniform illumination (DC component) summed to a non-uniform pattern (with positive and negative intensities). The presence of the DC component limits the advantage of using structured light. Due to the inherent tissue response, the DC component is much less attenuated than the higher spatial frequencies of the pattern. Therefore, the fluorescence images obtained with high spatial frequencies illumination are very similar to those obtained with uniform illuminations and the reconstruction quality is slightly improved by the use of patterned illumination. To alleviate this problem, the so-called virtual source pattern (VSP) method, which allows for considering any source pattern, has been introduced. Implementing this method, virtual source patterns with no DC components are shown to improve significantly the reconstruction. In particular, patterns belonging to 2-D wavelet bases offer the best performance. It is worth outlining that the VSP method has the ability to reject the undesirable sources of light. This is a key feature in experimental scenarios for which the illumination of the object is unavoidably corrupted by some light background. Even though the results of this paper have been verified in FDOT experiments, we 
believe that many aspects of the results and discussion can be translated to optical system based on photon migration, such as diffuse optical tomography and lifetime tomography. In future work, we will investigate the benefit of the proposed method in small animal imaging.

\section{Appendix}

\section{Appendix A.1. Integration kernel of the forward operator}

The retained component $m_{k}$ can be expressed as the projection of the measured image $\boldsymbol{m}$ onto the detection pattern $\boldsymbol{d}_{k}$. Generally speaking we have:

$$
m_{k}=\left\langle\boldsymbol{m}, \boldsymbol{d}_{k}\right\rangle_{\Sigma^{\mathrm{m}}}
$$

Substituting $\boldsymbol{m}=\mathcal{M} \phi^{\mathrm{f}}$ in the previous equation lead to:

$$
\begin{aligned}
m_{k} & =\left\langle\mathcal{M} \phi^{\mathrm{f}}, \boldsymbol{d}_{k}\right\rangle_{\Sigma^{\mathrm{m}}} \\
& =\left\langle\mathcal{M} \mathcal{G}^{\mathrm{f}}\left[\phi^{\mathrm{e}} f\right], \boldsymbol{d}_{k}\right\rangle_{\Sigma^{\mathrm{m}}} .
\end{aligned}
$$

By definition of the adjoint operator, we have:

$$
\begin{aligned}
m_{k} & =\left\langle\mathcal{G}^{f *}\left[\phi^{\mathrm{e}} f\right], \mathcal{M}^{*} \boldsymbol{d}_{k}\right\rangle_{\partial \Omega^{\mathrm{m}}} \\
& =\left\langle\phi^{\mathrm{e}} f, \mathcal{G}^{\mathrm{f} *}\left[\mathcal{M}^{*} \boldsymbol{d}_{k}\right]\right\rangle_{\Omega} .
\end{aligned}
$$

Isolating $f$ that is the quantity of interest, we obtain

$$
\begin{aligned}
m_{k} & =\left\langle f, \overline{\phi^{\mathrm{e}}} \mathcal{G}^{\mathrm{f} *}\left[\mathcal{M}^{*} \boldsymbol{d}_{k}\right]\right\rangle_{\Omega} \\
& =\left\langle\phi^{\mathrm{e}} \overline{\mathcal{G}^{\mathrm{f} *}\left[\mathcal{M}^{*} \boldsymbol{d}_{k}\right]}, f\right\rangle_{\Omega}
\end{aligned}
$$

and finally

$$
m_{k}=\left\langle\mathcal{G}^{\mathrm{e}}[\mathcal{I} \boldsymbol{s}] \overline{\mathcal{G}^{\mathrm{f} *}\left[\mathcal{M}^{*} \boldsymbol{d}_{k}\right]}, f\right\rangle_{\Omega}
$$

\section{Appendix A.2. Derivation of Matrix $\mathbf{T}$}

Any wavelet basis is associated to a pair of high-pass and low pass filters, denoted $\boldsymbol{h}$ and $\boldsymbol{g}$, respectively. We denote $L$ the length of the filters, $M_{\mathrm{V}}$ the number of desired wavelets along the first direction (vertical), and $M_{\mathrm{H}}$ the number of desired wavelets along the second direction (horizontal). The matrix $\boldsymbol{T}$, which is of size $3 M_{\mathrm{V}} M_{\mathrm{H}} \times 4 M_{\mathrm{V}} M_{\mathrm{H}}$, can be derived from the pair of filters following the steps:

(i) Resizing 1-D filters. We first build two filters of size $M_{\mathrm{V}} \times 1$ denoted $\boldsymbol{h}^{\mathrm{V}}$ and $\boldsymbol{g}^{\mathrm{V}}$. If $L<M_{\mathrm{V}}$, the filters are obtained by padding $\boldsymbol{h}$ and $\boldsymbol{g}$ with zeros so as to get the desired length. If $L>M_{\mathrm{V}}$, the filters are 'periodised' with a period $M_{\mathrm{V}}$, i.e. $h^{\mathrm{V}}[i]=\sum_{k=0} h\left[i+k M_{\mathrm{V}}\right]$ and $g^{\mathrm{V}}[i]=\sum_{k=0} g\left[i+k M_{\mathrm{V}}\right]$ are considered. The same way, we build $\boldsymbol{h}^{\mathrm{H}}$ and $\boldsymbol{g}^{\mathrm{H}}$ that are two filters of size $M_{\mathrm{H}} \times 1$. 
(ii) Computing 2-D filters. The vertical, horizontal and diagonal 2-D filters, denoted $G^{\mathrm{V}}, G^{\mathrm{H}}$, and $G^{\mathrm{D}}$, respectively, are obtained by:

$$
\boldsymbol{G}^{\mathrm{V}}=\boldsymbol{h}^{\mathrm{V}}\left(\boldsymbol{g}^{\mathrm{H}}\right)^{\mathrm{T}}, \boldsymbol{G}^{\mathrm{H}}=\boldsymbol{g}^{\mathrm{V}}\left(\boldsymbol{h}^{\mathrm{H}}\right)^{\mathrm{T}} \text {, and } \boldsymbol{G}^{\mathrm{D}}=\boldsymbol{g}^{\mathrm{V}}\left(\boldsymbol{g}^{\mathrm{H}}\right)^{\mathrm{T}} \text {. }
$$

(iii) Circularly shifting 2-D filters. The 2-D filters obtained at the previous step must be circularly shifted by multiples of 2 . We denote $\boldsymbol{G}_{i, j}$ the 2 -D filter resulting from a circular shift of $G$ by $i$ elements along its first dimension and by $j$ elements along its second dimension. The following shifted version of $G^{\mathrm{V}}, \boldsymbol{G}^{\mathrm{H}}$, and $\boldsymbol{G}^{\mathrm{D}}$ are required:

$$
\boldsymbol{G}_{2 i, 2 j}^{\mathrm{V}}, \boldsymbol{G}_{2 i, 2 j}^{\mathrm{H}} \text {, and } \boldsymbol{G}_{2 i, 2 j}^{\mathrm{D}} \text { for }(i, j) \in\left\{0, \ldots, M_{\mathrm{V}}-1\right\} \times\left\{0, \ldots, M_{\mathrm{H}}-1\right\} \text {. }
$$

(iv) Vectorising. Each of the shifted 2-D filter is vectorised to provide a row of $\boldsymbol{T}$. Noting $\operatorname{vect}(\boldsymbol{G})$ the vectorised version of $\boldsymbol{G}$, we have:

$$
\boldsymbol{T}=\left(\begin{array}{c}
\operatorname{vect}\left(\boldsymbol{G}_{0,0}^{\mathrm{V}}\right)^{\mathrm{T}} \\
\operatorname{vect}\left(\boldsymbol{G}_{0,0}^{\mathrm{H}}\right)^{\mathrm{T}} \\
\operatorname{vect}\left(\boldsymbol{G}_{0,0}^{\mathrm{D}}\right)^{\mathrm{T}} \\
\operatorname{vect}\left(\boldsymbol{G}_{0,1}^{\mathrm{V}}\right)^{\mathrm{T}} \\
\operatorname{vect}\left(\boldsymbol{G}_{0,1}^{\mathrm{H}}\right)^{\mathrm{T}} \\
\operatorname{vect}\left(\boldsymbol{G}_{0,1}^{\mathrm{D}}\right)^{\mathrm{T}} \\
\vdots \\
\operatorname{vect}\left(\boldsymbol{G}_{M_{\mathrm{V}}-1, M_{\mathrm{H}}-1}^{\mathrm{V}}\right)^{\mathrm{T}} \\
\operatorname{vect}\left(\boldsymbol{G}_{M_{\mathrm{V}}-1, M_{\mathrm{H}}-1}^{\mathrm{H}}\right)^{\mathrm{T}} \\
\operatorname{vect}\left(\boldsymbol{G}_{M_{\mathrm{V}}-1, M_{\mathrm{H}}-1}^{\mathrm{D}}\right)^{\mathrm{T}}
\end{array}\right)
$$

Example In the Haar case, we have the following low-pass and hight-pass filters:

$$
\boldsymbol{h}=\frac{1}{\sqrt{2}}[1,1]^{\mathrm{T}} \text { and } \boldsymbol{g}=\frac{1}{\sqrt{2}}[1,-1]^{\mathrm{T}} .
$$

Choosing $M_{\mathrm{V}}=2$ and $M_{\mathrm{H}}=1$, we build (step i):

$$
\begin{aligned}
\boldsymbol{h}^{\mathrm{V}}=\frac{1}{\sqrt{2}}[1,1,0,0]^{\mathrm{T}}, \boldsymbol{g}^{\mathrm{V}}=\frac{1}{\sqrt{2}}[1,-1,0,0]^{\mathrm{T}}, \\
\boldsymbol{h}^{\mathrm{H}}=\frac{1}{\sqrt{2}}[1,1]^{\mathrm{T}}, \boldsymbol{g}^{\mathrm{H}}=\frac{1}{\sqrt{2}}[1,-1]^{\mathrm{T}} .
\end{aligned}
$$

From the previous four 1-D filters, we build the following three 2-D filters (step ii):

$$
\boldsymbol{G}^{\mathrm{V}}=\left(\begin{array}{cc}
1 & -1 \\
1 & -1 \\
0 & 0 \\
0 & 0
\end{array}\right), \boldsymbol{G}^{\mathrm{H}}=\left(\begin{array}{cc}
1 & 1 \\
-1 & -1 \\
0 & 0 \\
0 & 0
\end{array}\right), \boldsymbol{G}^{\mathrm{D}}=\left(\begin{array}{cc}
1 & -1 \\
-1 & 1 \\
0 & 0 \\
0 & 0
\end{array}\right)
$$


Next, we have to shift the 2-D filters (step iii):

$$
\begin{gathered}
\boldsymbol{G}_{0,0}^{\mathrm{V}}=\left(\begin{array}{cc}
1 & -1 \\
1 & -1 \\
0 & 0 \\
0 & 0
\end{array}\right), \boldsymbol{G}_{0,0}^{\mathrm{H}}=\left(\begin{array}{cc}
1 & 1 \\
-1 & -1 \\
0 & 0 \\
0 & 0
\end{array}\right), \boldsymbol{G}_{0,0}^{\mathrm{D}}=\left(\begin{array}{cc}
1 & -1 \\
-1 & 1 \\
0 & 0 \\
0 & 0
\end{array}\right), \\
\boldsymbol{G}_{1,0}^{\mathrm{V}}=\left(\begin{array}{cc}
0 & 0 \\
0 & 0 \\
1 & -1 \\
1 & -1
\end{array}\right), \boldsymbol{G}_{1,0}^{\mathrm{H}}=\left(\begin{array}{cc}
0 & 0 \\
0 & 0 \\
1 & 1 \\
-1 & -1
\end{array}\right), \boldsymbol{G}_{1,0}^{\mathrm{D}}=\left(\begin{array}{cc}
0 & 0 \\
0 & 0 \\
1 & -1 \\
-1 & 1
\end{array}\right) .
\end{gathered}
$$

Vectorising the previous six matrices, we obtain the matrix $\boldsymbol{T}$ as given in (20) (step iv).

\section{Acknowledgment}

This work was partly supported by Royal Society International Joint Project 2009/R2, Cariplo Foundation (Grant 2009-2626) and MIUR under the project Futuro in Ricerca (prot. RBFR08XH0H 002).

\section{References}

\section{References}

Arridge, S. R. and Schotland, J. C. (2009). Optical tomography: forward and inverse problems. Inverse Problems, 25(12):123010 (59pp).

Baritaux, J.-C., Hassler, K., and Unser, M. (2010). An efficient numerical method for general $l_{p}$ regularization in fluorescence molecular tomography. Medical Imaging, IEEE Transactions on, 29(4):1075 -1087.

Bassi, A., D'Andrea, C., Valentini, G., Cubeddu, R., and Arridge, S. (2008). Temporal propagation of spatial information in turbid media. Opt. Lett., 33(23):2836-2838.

Bassi, A., D'Andrea, C., Valentini, G., Cubeddu, R., and Arridge, S. (2009). Detection of inhomogeneities in diffusive media using spatially modulated light. Opt. Lett., 34(14):2156-2158.

Bassi, A., Farina, A., D’Andrea, C., Pifferi, A., Valentini, G., and Cubeddu, R. (2007). Portable, large-bandwidth time-resolved system for diffuse optical spectroscopy. Opt. Express, 15(22):14482-14487.

Bélanger, S., Abran, M., Intes, X., Casanova, C., and Lesage, F. (2010). Real-time diffuse optical tomography based on structured illumination. Journal of Biomedical Optics, 15(1):016006.

Carlson, A., Crilly, P., and Rutlege, J. (2002). Communication systems. McGraw-Hill, 4th edition.

Chen, J., Venugopal, V., Lesage, F., and Intes, X. (2010). Time-resolved diffuse optical tomography with patterned-light illumination and detection. Opt. Lett., $35(13): 2121-2123$.

Cherry, S. R. (2004). In vivo molecular and genomic imaging: new challenges for imaging physics. Physics in Medicine and Biology, 49(3):R13. 
Cuccia, D. J., Bevilacqua, F., Durkin, A. J., and Tromberg, B. J. (2005). Modulated imaging: quantitative analysis and tomography of turbid media in the spatialfrequency domain. Opt. Lett., 30(11):1354-1356.

D'Andrea, C., Ducros, N., Bassi, A., Arridge, S., and Valentini, G. (2010). Fast 3d optical reconstruction in turbid media using spatially modulated light. Biomed. Opt. Express, 1(2):471-481.

Ducros, N., Bassi, A., Valentini, G., Schweiger, M., Arridge, S., and D'Andrea, C. (2011). Multiple-view fluorescence optical tomography reconstruction using compression of experimental data. Opt. Lett., 36(8):1377-1379.

Ducros, N., D'andrea, C., Valentini, G., Rudge, T., Arridge, S., and Bassi, A. (2010a). Full-wavelet approach for fluorescence diffuse optical tomography with structured illumination. Opt. Lett., 35(21):3676-3678.

Ducros, N., Silva, A. D., Dinten, J.-M., Seelamantula, C. S., Unser, M., and Peyrin, F. (2010b). A time-domain wavelet-based approach for fluorescence diffuse optical tomography. Medical Physics, 37(6):2890-2900.

Ducros, N., Silva, A. D., Herve, L., Dinten, J.-M., and Peyrin, F. (2009). A comprehensive study of the use of temporal moments in time-resolved diffuse optical tomography: part ii. three-dimensional reconstructions. Physics in Medicine and Biology, 54(23):7107-7119.

Dutta, J., Ahn, S., Joshi, A. A., and Leahy, R. M. (2010). Illumination pattern optimization for fluorescence tomography: theory and simulation studies. Physics in Medicine and Biology, 55(10):2961.

Herv, L., Koenig, A., Da Silva, A., Berger, M., Boutet, J., Dinten, J., Peltié, P., and Rizo, P. (2007). Noncontact fluorescence diffuse optical tomography of heterogeneous media. Applied Optics, 46(22):4896-4906.

Ishimaru, A. (1977). Theory and application of wave propagation and scattering in random media. Proceedings of the IEEE, 65(7):1030-1061.

Joshi, A., Bangerth, W., Hwang, K., Rasmussen, J., and Sevick-Muraca, E. M. (2006a). Plane-wave fluorescence tomography with adaptive finite elements. Opt. Lett., 31(2):193-195.

Joshi, A., Bangerth, W., and Sevick-Muraca, E. M. (2006b). Non-contact fluorescence optical tomography with scanning patterned illumination. Opt. Express, 14(14):6516-6534.

Klose, A. D. and Pschinger, T. (2011). Excitation-resolved fluorescence tomography with simplified spherical harmonics equations. Physics in Medicine and Biology, $56(5): 1443$

Koenig, A., Herve, L., Josserand, V., Berger, M., Boutet, J., Silva, A. D., Dinten, J.-M., Peltie, P., Coll, J.-L., and Rizo, P. (2008). In vivo mice lung tumor followup with fluorescence diffuse optical tomography. Journal of Biomedical Optics, 13(1):011008.

Konecky, S. D., Mazhar, A., Cuccia, D., Durkin, A. J., Schotland, J. C., and Tromberg, B. J. (2009). Quantitative optical tomography of sub-surface heterogeneities using spatially modulated structured light. Opt. Express, 17(17):14780-14790.

Konecky, S. D., Panasyuk, G. Y., Lee, K., Markel, V., Yodh, A. G., and Schotland, J. C. (2008). Imaging complex structures with diffuse light. Opt. Express, 16(7):5048-5060. 
Kumar, A. T. N., Raymond, S. B., Dunn, A. K., Bacskai, B. J., and Boas, D. A. (2008). A time domain fluorescence tomography system for small animal imaging. Medical Imaging, IEEE Transactions on, 27(8):1152-1163.

Lukic, V., Markel, V. A., and Schotland, J. C. (2009). Optical tomography with structured illumination. Opt. Lett., 34(7):983-985.

Mallat, S. (1989). A theory for multiresolution signal decomposition: the wavelet representation. Pattern Analysis and Machine Intelligence, IEEE Transactions on, 11(7):674-693.

Markel, V. A., Mital, V., and Schotland, J. C. (2003). Inverse problem in optical diffusion tomography. iii. inversion formulas and singular-value decomposition. $J$. Opt. Soc. Am. A, 20(5):890-902.

Markel, V. A. and Schotland, J. C. (2004). Symmetries, inversion formulas, and image reconstruction for optical tomography. Phys. Rev. E, 70(5):056616.

Milstein, A. B., Oh, S., Webb, K. J., Bouman, C. A., Zhang, Q., Boas, D. A., and Millane, R. P. (2003). Fluorescence optical diffusion tomography. Appl. Opt., 42(16):3081-3094.

Montet, X., Figueiredo, J.-L., Alencar, H., Ntziachristos, V., Mahmood, U., and Weissleder, R. (March 2007). Tomographic fluorescence imaging of tumor vascular volume in mice1. Radiology, 242(3):751-758.

Neil, M. A. A., Juskaitis, R., and Wilson, T. (1997). Method of obtaining optical sectioning by using structured light in a conventional microscope. Opt. Lett., 22(24):1905-1907.

Ntziachristos, V., Bremer, C., and Weissleder, R. (2003). Fluorescence imaging with near-infrared light: new technological advances that enable in vivo molecular imaging. European Radiology, 13(1):195-208.

Ntziachristos, V., Ripoll, J., Wang, L., and Weissleder, R. (2005). Looking and listening to light: the evolution of whole-body photonic imaging. Nature Biotechnology, 23(3):313-320.

Panasyuk, G. Y., Wang, Z.-M., Schotland, J. C., and Markel, V. A. (2008). Fluorescent optical tomography with large data sets. Opt. Lett., 33(15):1744-1746.

Ripoll, J. (2010). Hybrid fourier-real space method for diffuse optical tomography. Opt. Lett., 35(5):688-690.

Rudge, T. J., Soloviev, V. Y., and Arridge, S. R. (2010). Fast image reconstruction in fluoresence optical tomography using data compression. Opt. Lett., 35(5):763-765.

Schweiger, M. and Arridge, S. R. (2010). TOAST package, http: //web4.cs.ucl. ac. uk/research/vis/toast/.

Song, X., Pogue, B. W., Jiang, S., Doyley, M. M., Dehghani, H., Tosteson, T. D., and Paulsen, K. D. (2004). Automated region detection based on the contrast-to-noise ratio in near-infrared tomography. Appl. Opt., 43(5):1053-1062.

Srinivasan, V., Liu, H. C., and Halioua, M. (1984). Automated phase-measuring profilometry of 3-d diffuse objects. Appl. Opt., 23(18):3105-3108.

Unser, M. and Blu, T. (2003). Wavelet theory demystified. Signal Processing, IEEE Transactions on [see also Acoustics, Speech, and Signal Processing, IEEE Transactions on], 51(2):470-483. 
Wang, Z.-M., Panasyuk, G. Y., Markel, V. A., and Schotland, J. C. (2005). Experimental demonstration of an analytic method for image reconstruction in optical diffusion tomography with large data sets. Opt. Lett., 30(24):3338-3340. 